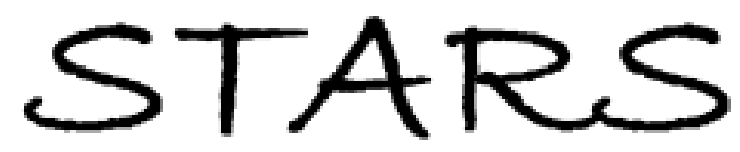

University of Central Florida

STARS

\title{
Effects of the final-state electron-ion interactions on the fully differential cross sections for heavy-particle-impact ionization of helium
}
A. L. Harris
D. H. Madison
J. L. Peacher
M. Foster
K. Bartschat

See next page for additional authors

Find similar works at: https://stars.library.ucf.edu/facultybib2000

University of Central Florida Libraries http://library.ucf.edu

This Article is brought to you for free and open access by the Faculty Bibliography at STARS. It has been accepted for inclusion in Faculty Bibliography 2000s by an authorized administrator of STARS. For more information, please contact STARS@ucf.edu.

\section{Recommended Citation}

Harris, A. L.; Madison, D. H.; Peacher, J. L.; Foster, M.; Bartschat, K.; and Saha, H. P., "Effects of the finalstate electron-ion interactions on the fully differential cross sections for heavy-particle-impact ionization of helium" (2007). Faculty Bibliography 2000s. 7211.

https://stars.library.ucf.edu/facultybib2000/7211

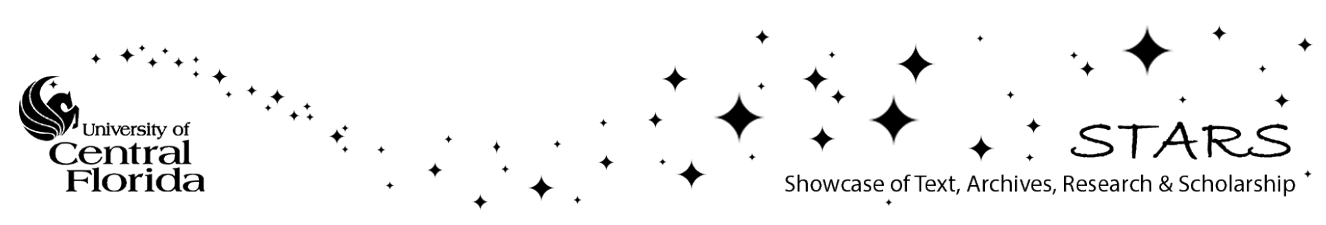


Authors

A. L. Harris, D. H. Madison, J. L. Peacher, M. Foster, K. Bartschat, and H. P. Saha 


\title{
Effects of the final-state electron-ion interactions on the fully differential cross sections for heavy-particle-impact ionization of helium
}

\author{
A. L. Harris, ${ }^{1}$ D. H. Madison, ${ }^{1}$ J. L. Peacher, ${ }^{1}$ M. Foster, ${ }^{2}$ K. Bartschat,${ }^{3}$ and H. P. Saha ${ }^{4}$ \\ ${ }^{1}$ University of Missouri-Rolla, Rolla, Missouri 65409, USA \\ ${ }^{2}$ Los Alamos National Laboratory, Los Alamos, New Mexico 87545, USA \\ ${ }^{3}$ Drake University, Des Moines, Iowa 50311, USA \\ ${ }^{4}$ University of Central Florida, Orlando, Florida 32816, USA
}

(Received 8 December 2006; revised manuscript received 26 February 2007; published 26 March 2007)

\begin{abstract}
Three-dimensional fully differential cross sections for heavy-particle-impact ionization of helium are examined. Previously, the three-body distorted-wave (3DW) model has achieved good agreement with experiment in the scattering plane for small momentum transfers, but poor agreement for large momentum transfers. Poor agreement was also observed outside the scattering plane for all momentum transfers. In particular, the 3DW calculations predicted cross sections that were too small both perpendicular to the scattering plane and for large momentum transfers. The important unanswered question concerns the physical effects that cause the significant disagreement between experiment and theory. In previous works, the role of the projectile-ion interaction has been examined. Although the importance of exchange between the ejected electron and the residual bound electrons has been well established, and frequently studied, for electron-impact ionization, the importance of this effect has not been examined for heavy-particle scattering. In this paper we examine the role of this effect for heavy-particle scattering.
\end{abstract}

DOI: 10.1103/PhysRevA.75.032718

PACS number(s): $34.50 .-\mathrm{s}$

\section{INTRODUCTION}

Experimental results for heavy-particle-impact ionization of helium have shown that what was once believed to be a solved problem is still not fully understood [1]. These experiments, performed on a cold-target-recoil-ion-momentum spectrometer (COLTRIMS) apparatus [2], measured fully differential cross sections (FDCSs) in the scattering plane, as well as out of the scattering plane. Agreement between experiment and theory in the scattering plane has been good for high-energy electron-impact ionization for many years $[3,4]$, so it was surprising to see large discrepancies out of the scattering plane. In particular, the $100 \mathrm{MeV} / \mathrm{amu} \mathrm{C}^{6+}$ experimental results show a distinct, ringlike feature out of the scattering plane, while theory fails to predict much structure $[1,4-6]$. Also surprising is the fact that agreement between experiment and theory becomes better as the incident projectile energy decreases [3]. This is contrary to intuition and traditional training, which would suggest that agreement would become better as projectile energy increases.

Recently, similar results have also been observed for $(e, 2 e)$ experiments when looking outside the scattering plane. Van Boeyen et al. [7] measured the FDCS for electron-impact ionization of magnesium in the $3 s^{2}$ state for a $45^{\circ}$ half-angle cone centered on the momentum transfer direction, and found significantly larger cross sections perpendicular to the scattering plane as compared to in plane. Also, experiments performed by Dürr et al. $[8,9]$ found significant out-of-plane cross sections for $102 \mathrm{eV}$ electronimpact ionization of helium. Theoretical calculations for the $\mathrm{Mg}$ experiment, performed by Foster et al. [5], showed that a distorted-wave treatment of the projectile electron provided much larger out-of-plane cross sections than a Coulombwave treatment for electron-magnesium scattering. For the helium experiment, the second-order distorted-wave Born approximation was in very good agreement with experiment, so the out-of-plane structure was attributed to multiple collisions between the projectile and the atom or residual ion. While these experiments are primarily viewed as three-body problems, a similar effect has been seen in a purely fourbody problem. For double ionization of helium, Voitkiv et al. [10] showed that the nuclear-nuclear interaction can play an important role in the shape of the FDCS.

There have been several suggestions concerning the source of the discrepancy between the out-of-plane experiment and theory. Olson and co-workers [11,12] suggested that the structure might be attributed to experimental resolution. A detailed analysis of the effect of experimental resolution will be published soon by an experimental group [13]. It has also been proposed that the failure of theory at high energy might be caused by the theory's final-state three-body double continuum Coulomb wave function breaking down when all particles are close together [6]. Although most of the studies have concentrated on the projectile wave function, Madison et al. [3] showed that it was very important to treat the ejected electron as a distorted wave to get improved agreement with experiment in the scattering plane. Coulombwave treatments for this part of the final-state wave function failed to yield proper magnitudes for the binary and recoil peaks. In the scattering plane, the three-body distorted-wave (3DW) model results are in good agreement with experiment for small momentum transfers, but the agreement deteriorates with increasing momentum transfer. One of the finalstate effects that has not been examined is exchange between the low-energy ejected electron and the residual bound-state electrons. For electron-impact ionization, the importance of this effect was established several years ago, and it has been frequently studied [14-17]. However, it has not been examined for heavy-particle ionization. In this work, we investigate the role of exchange between the ejected and bound electrons for heavy-particle ionization by using continuum Hartree-Fock $[14,15]$ and $R$-matrix [18] wave functions for the ejected electron. 


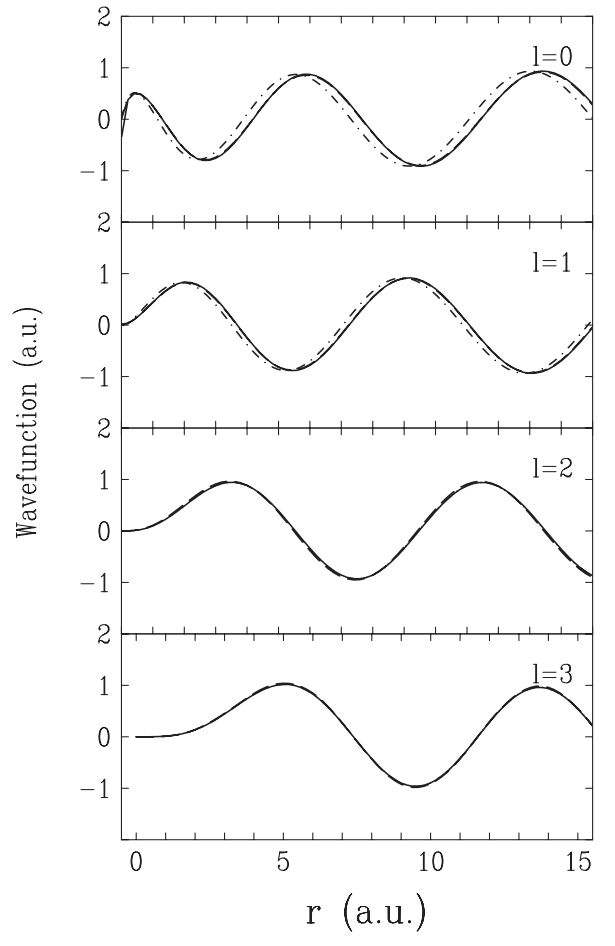

FIG. 1. $l=0-3$ partial waves for the ionized electron with an energy of $17.5 \mathrm{eV}$ obtained from distorted-wave (dash dotted), continuum Hartree-Fock (solid), and three-state $R$-matrix (dashed) calculations.

\section{THEORY}

The theoretical approach we use for this study is the 3DW model. Details about the 3DW approach can be found in Refs. [3,4], so we give only a brief overview to point out the important components of the theory for this work. The $3 \mathrm{DW}$ transition matrix ( $T$ matrix) is given by

$$
T_{f i}^{3 D W}=\left\langle(\mathrm{CW})_{f} \chi_{\text {eject }} C_{\text {proj-eject }}|V| \psi_{\text {active }}(\mathrm{PW})_{i}\right\rangle
$$

Here $\psi_{\text {active }}$ is the initial bound-state wave function for the active electron, $(\mathrm{PW})_{i}$ is an initial-state plane wave for the projectile, $(\mathrm{CW})_{f}$ is a final-state Coulomb wave for the projectile for a Coulomb field of unit charge, $\chi_{\text {eject }}$ is the wave function for the ejected electron, $C_{\text {proj-eject }}$ is the Coulomb interaction between the projectile and the ejected electron, and $V$ is the perturbation.

In the 3DW T matrix of Eq. (1), the final-state Coulomb interaction between the projectile and ejected electrons $C_{\text {proj-eject }}$ [normally called postcollision interaction (PCI)] is included in the approximation for the final-state wave function. The important point to note is that any physics included directly in the wave function is included to all orders of perturbation theory for the projectile-ejected-electron subsystem, and therefore the 3DW theory also has PCI included to all orders of perturbation theory. In the standard 3DW approach, $\chi_{\text {eject }}$ is a final-state distorted wave which is a solution of the Schrödinger equation

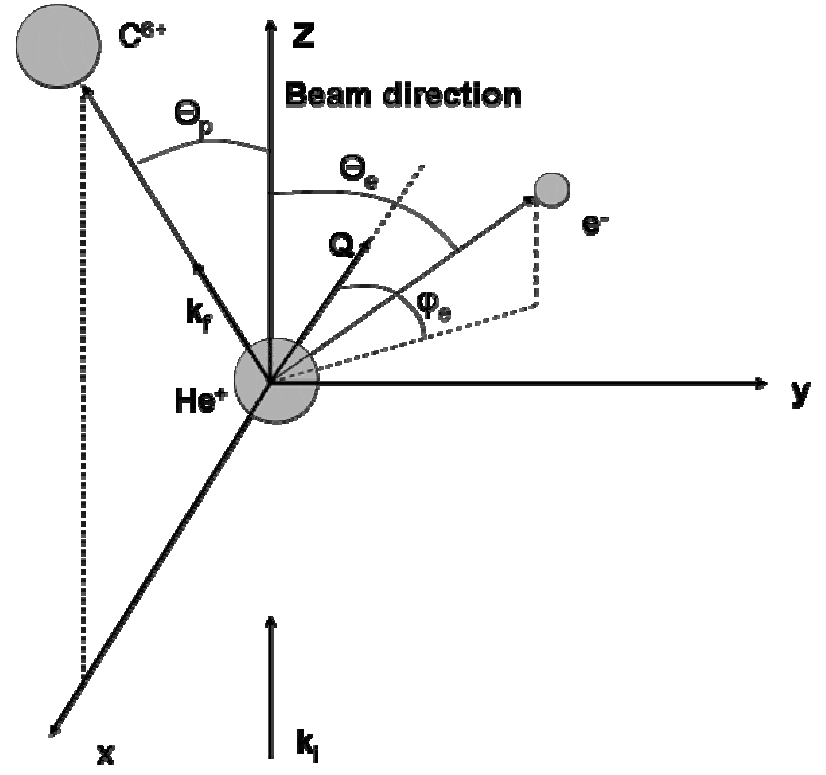

FIG. 2. Coordinate system for the collision. The beam direction is the $z$ axis. The projectile scatters at an angle of $\theta_{p}$ and the ejected electron observation angles are $\theta_{e}$ and $\varphi_{e}$. The scattering plane is the $x z$ plane $\left(\varphi_{e}=0^{\circ}\right)$ and the perpendicular plane is the $y z$ plane $\left(\varphi_{e}=90^{\circ}\right)$.

$$
\left(T+U_{\text {ion }}-k_{\text {eject }}^{2}\right) \chi_{\text {eject }}^{D W}=0 .
$$

Here $T$ is the kinetic energy operator, $U_{i o n}$ is the distorting potential for the ion, and $k_{\text {eject }}^{2}$ is the energy of the ejected electron. The Schrödinger equation (2) does not contain the effects of exchange between the ejected electron and residual bound electrons, which is known to be very important for ejected electrons with an energy of less than about $50 \mathrm{eV}$ $[15,16]$. To investigate the importance of this exchange for heavy-particle ionization we use two different wave functions, in addition to the normal distorted waves, for $\chi_{\text {eject }}$, both of which contain this exchange effect to numerical accuracy. The first wave function we use is the Hartree-Fock (HF) continuum wavefunction [14] $\chi_{\text {eject }}^{H F}$ and we label results using this wave function 3DW-HF. The second one we use is an $R$-matrix (RM) (close-coupling) wave function [18] $\chi_{\text {eject }}^{R M}$, and we label results using this wave function as 3DW-RM.

The distorted waves are calculated using a partial-wave expansion and the procedure we use to evaluate HartreeFock and $R$-matrix results is to replace the first several partial waves with either continuum Hartree-Fock (3DW-HF) or three-state $R$-matrix (3DW-RM) waves. After roughly three to four partial waves, the Hartree-Fock and $R$-matrix waves become nearly identical to the distorted waves, and the rest of the calculation is done with standard distorted waves. The convergence of the waves is shown in Fig. 1. Other than this, no further changes need to be made to the 3DW theory.

\section{RESULTS}

The coordinate system is shown in Fig. 2 with the $z$ axis oriented along the incident beam direction. The projectile scatters at an angle $\theta_{p}$ in the $+x$ direction, which is on the 


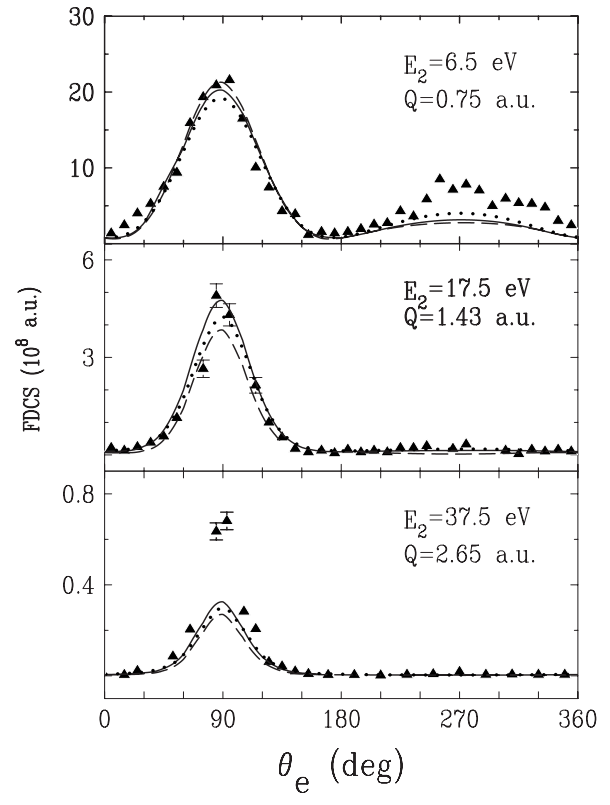

FIG. 3. FDCSs in the scattering plane $\left(\phi_{e}=90^{\circ}\right)$ for $100 \mathrm{MeV} / \mathrm{amu} \mathrm{C}^{6+}$ single ionization of helium with ionized electron energies of $6.5,17.5$, and $37.5 \mathrm{eV}$. The momentum transfers are $0.75,1.43$, and 2.65 a.u., respectively. The dotted line is the 3DW calculation, solid is 3DW-HF, dashed is 3DW-RM, and the absolute experimental results are the triangles.

order of microradians, and the ionized electron comes out at a location labeled by $\theta_{e}$ and $\varphi_{e}$. It should be noted that this coordinate system is not the standard spherical coordinate system. Here, $\varphi_{e}$ ranges from $0^{\circ}$ to $90^{\circ}$, while $\theta_{e}$ ranges from $0^{\circ}$ to $360^{\circ}$. While this covers only half of the space, the other half is known automatically by symmetry. The scattering plane, defined by the incident and final momentum vectors of the projectile, is the $x z$ plane, or the $\varphi_{e}=0^{\circ}$ plane, and the perpendicular plane is the $y z$ plane, or the $\varphi_{e}=90^{\circ}$ plane. The momentum transfer direction, defined as $\mathbf{Q}=\mathbf{k}_{i}-\mathbf{k}_{f}$, lies nearly on the negative $x$ axis due to high projectile mass and energy and small scattering angle.

Absolute experimental measurements of the FDCSs [3] are presented in the center-of-mass system. Atomic units are used unless otherwise noted. In Fig. 3, these measurements are compared to the 3DW, 3DW-HF, and 3DW-RM predictions for three different ejected electron energies and momentum transfers in the scattering plane.

The large peak at small $\theta_{e}$ angles is normally called the binary peak since it is located near the angle that would result from a classical binary collision between the projectile and a free electron at rest. The smaller peak at large $\theta_{e}$ angles is called the recoil peak, and it is attributed to a two-collision process in which the atomic electron first encounters a binary collision with the projectile followed by a $180^{\circ}$ secondary backscattering from the residual ion. In the binary peak, the 3DW-HF results are in better agreement with the experimental data than the 3DW for all cases, while the 3DW-RM method shows improved agreement for the $6.5 \mathrm{eV}$ case, but not for the higher energies and momentum transfers. For the $37.5 \mathrm{eV}$ case, the improvement seen for the $3 \mathrm{DW}-\mathrm{HF}$ case is small compared to the large discrepancy between experiment

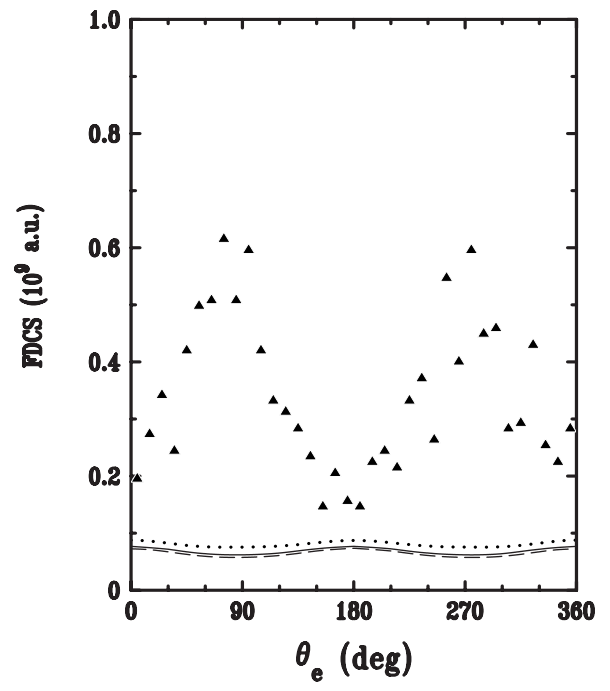

FIG. 4. FDCSs in the perpendicular plane $\left(\varphi_{e}=90^{\circ}\right)$ for $100 \mathrm{MeV} / \mathrm{amu} \mathrm{C}^{6+}$ single ionization of helium with ionized electron energy of $6.5 \mathrm{eV}$ and momentum transfer of $0.75 \mathrm{a} . \mathrm{u}$. Labels are the same as in Fig. 3.

and theory. This observation is consistent with intuition, which would suggest that exchange between the continuum electron and ion should become less important with increasing ejected-electron energy, while the large disagreement between experiment and theory for the larger momentum transfers is probably caused by an inadequate treatment of the projectile-ion interaction. One particularly interesting feature can be seen in the $17.5 \mathrm{eV}$ case where both the 3DW and 3DW-HF calculations give a small maximum in the recoil lobe, while the 3DW-RM method gives a minimum. Overall, in-plane exchange between the ejected and bound electrons tends to improve agreement with experiment for the binary peak and worsen agreement for the recoil peak. Since the recoil peak is a backscattering of the ejected electron from the ion, it appears that exchange with the bound electrons for this relatively close collision reduces the size of the cross sections. Obviously, there must be some other competing effect missing in the calculation which would increase the recoil peak.

Figure 4 contains the perpendicular plane results, which are available only at an ejected-electron energy of $6.5 \mathrm{eV}$ and $Q=0.75$ a.u. Unfortunately, the improvement that was seen in the scattering plane is not seen in the perpendicular plane. The 3DW-RM and 3DW-HF calculations differ very little from the 3DW calculation, and are actually in worse agreement with experiment. This indicates that the treatment of the ionized electron is not a significant part of the problem with theory out of the scattering plane. This observation further supports previous works, which have shown that at least part of the problem with the out-of-plane theory is an inadequate treatment of the projectile-ion interaction.

To gain a more quantitative idea of why agreement in the scattering plane is so different from the perpendicular plane, we have plotted the results for some intermediate planes in Fig. 5. Each panel is a plane determined by an angle $\varphi_{e}$ such that $\varphi_{e}=0^{0}$ corresponds to the scattering plane. It can be seen that as $\varphi_{e}$ increases the binary peak decreases for both ex- 


$$
\mathrm{E}_{2}=6.5 \mathrm{eV} Q=0.75 \text { a.u. }
$$

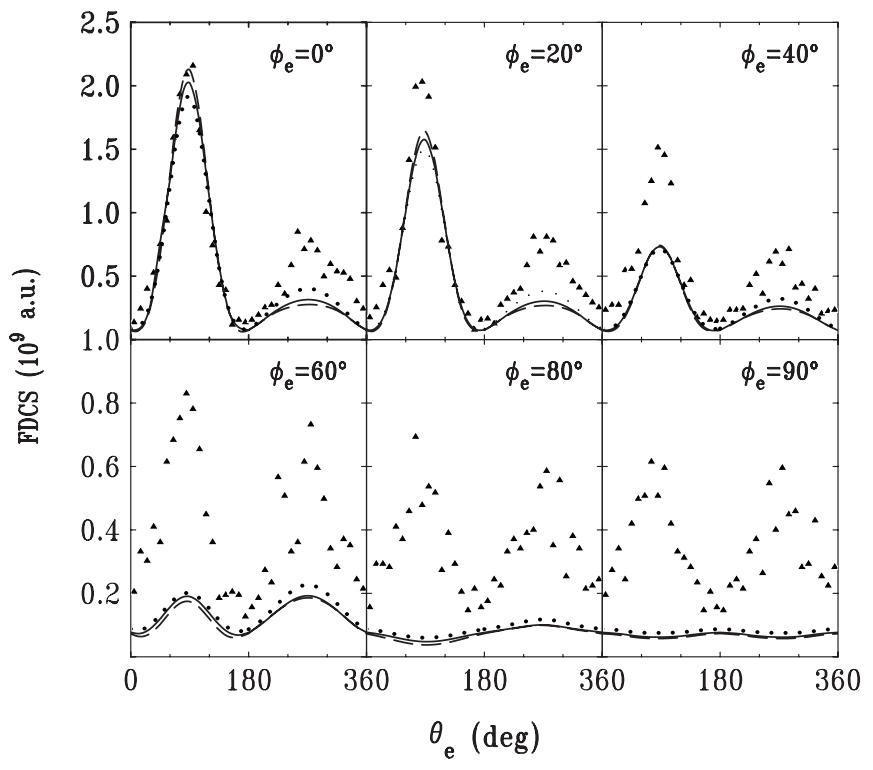

FIG. 5. FDCSs at different $\varphi_{e}$ angles. Labels are the same as in Fig. 3.

periment and theory. However, the drop in the binary peak is more drastic for theory, and the binary peak becomes a binary minimum at $\varphi_{e}=80^{\circ}$. In contrast, the experimental binary peak remains a peak and the angular location does not change.

On the recoil side, the magnitude of the theory stays roughly constant, but the peak begins to shift toward the beam direction at $\varphi_{e}=80^{\circ}$ and a small minimum appears at the angular location of the recoil peak. Similar to the experimental binary peak, the magnitude of the experimental recoil peak does not change dramatically for increasing $\varphi_{e}$ angles, and there is no shift in the angular location. Figure 5 clearly shows the deterioration of agreement between experiment and theory as one gets further from the scattering plane, and it is apparent that the effect of exchange between the ejected electron and bound electrons is not an important source of this disagreement.

\section{CONCLUSIONS}

For the scattering plane, a better treatment of the ejected electron creates an improved agreement with experiment for the binary peak, while for the perpendicular plane, not much change is seen at all. It is particularly evident that the electron-ion interaction is not an important cause of the disagreement between experiment and theory. Previous works have indicated that at least part of the problem with theory out of the scattering plane is an inadequate treatment of the close collisions between the projectile and ion $[5,6]$. However, it was not clear if this was the only problem, or if there could be additional problems associated with the need for a better treatment of the ejected-electron-ion interaction. This work demonstrates that the entire problem is probably due to the treatment of the projectile-ion interaction, and as Foster et al. [5] showed, at a minimum, a distorted-wave treatment of the projectile is needed. Although such a treatment has not yet been achieved, this work further emphasizes the need for the development of a distorted-wave theory for heavyparticle scattering.

\section{ACKNOWLEDGMENTS}

We gratefully acknowledge the support of the NSF under Grants No. PHY-045628 (D.M.) and No. PHY-0244470 (K.B.).
[1] M. Schulz, R. Moshammer, D. Fischer, H. Kollmus, D. H. Madison, S. Jones, and J. Ullrich, Nature (London) 422, 48 (2003).

[2] R. Moshammer, J. Ullrich, M. Unverzagt, W. Schmidt, P. Jardin, R. E. Olson, R. Mann, R. Dörner, V. Mergel, U. Buck, and H. Schmidt-Böcking, Phys. Rev. Lett. 73, 3371 (1994).

[3] D. H. Madison, M. Schulz, S. Jones, M. Foster, R. Moshammer, and J. Ullrich, J. Phys. B 35, 3297 (2002).

[4] M. Foster et al., J. Phys. B 37, 1565 (2004).

[5] M. Foster, J. L. Peacher, M. Schulz, D. H. Madison, Zhangjin Chen, and H. R. J. Walters, Phys. Rev. Lett. 97, 093202 (2006).

[6] D. H. Madison, D. Fischer, M. Foster, M. Schulz, R. Moshammer, S. Jones, and J. Ullrich, Phys. Rev. Lett. 91, 253201 (2003).

[7] R. W. van Boeyen, N. Watanabe, J. W. Cooper, J. P Doering, J. H. Moore, and M. A. Coplan, Phys. Rev. A 73, 032703 (2006).

[8] M. Dürr, C. Dimopoulou, A. Dorn, B. Najjari, I. Bray, D. V. Fursa, Zhangjin Chen, D. H. Madison, K. Bartschat, and J.
Ullrich, J. Phys. B 39, 4097 (2006).

[9] M. Dürr, C. Dimopoulou, B. Najjari, A. Dorn, and J. Ullrich, Phys. Rev. Lett. 96, 243202 (2006).

[10] A. B. Voitkiv, B. Najjari, and J. Ullrich, J. Phys. B 38, L107 (2005).

[11] R. E. Olson and J. Fiol, Phys. Rev. Lett. 95, 263203 (2005).

[12] J. Fiol, S. Otranto, and R. E. Olson, J. Phys. B 39, L285 (2006).

[13] M. Schulz (private communication).

[14] K. D. Winkler, D. H. Madison, and H. Saha, J. Phys. B 32, 4617 (1999).

[15] D. A. Biava, H. P. Saha, E. Engel, R. M. Dreizler, R. P. McEachran, M. A. Haynes, B. Lohmann, C. T. Whelan, and D. H. Madison, J. Phys. B 35, 293 (2002).

[16] D. A. Biava, K. Bartschat, H. P. Saha, and D. H. Madison, J. Phys. B 35, 5121 (2002).

[17] R. Panajotovic, J. Lower, E. Weigold, A. Prideaux, and D. H. Madison, Phys. Rev. A 73, 052701 (2006).

[18] K. Bartschat and P. G. Burke, J. Phys. B 20, 3191 (1987). 